\title{
LOS LIBROS DE CABALLERÍAS CASTELLANOS FRENTE AL SIGLO XXI \\ (a propósito de una nueva publicación) ${ }^{1}$
}

\author{
José Manuel Lucia Megias \\ Universidad Complutense de Madrid
}

Los libros de caballerías castellanos han merecido en los últimos años del siglo $\mathrm{xx}$ una atención que la crítica parecía haberles negado desde finales del XVIII. La comparación entre el Catálogo razonado de los libros de caballerias que hay en lengua castellana o portuguesa, hasta el año de 1800 que Pascual de Gayangos incluyó al inicio del primer tomo (y único publicado) de sus Libros de caballerias (Madrid, Rivadeneyra, 1857) y el Castilian Romances of Chivalry in the Sixteenth Century: A Bibliography de Daniel Eisenberg (Londres, Grant \& Cutler, 1979) o, si se prefiere, la excelente herramienta de estudio que está en el origen de este trabajo: la $B i$ bliografia de los libros de caballerías castellanos de Daniel Eisenberg y M. ${ }^{a}$ Carmen Marín Pina marca el camino de la rehabilitación de uno de los géneros narrativos más importantes de los siglos XVI y XVI, que había sido casi olvidado, a excepción de algunos títulos, bajo el peso de una lectura parcial (y falsa) de uno de los libros de caballerías más famosos: El Quijote de Miguel de Cervantes. Los estudios y ediciones, algo aislados dentro del panorama crítico internacional, de Henry Thomas, Edwin Place, Irving A. Leonard, Martín de Riquer, José Amezcua Gómez, Maxime Chevalier, Giuseppe di Stefano, Armando Durán, Bernard König, Juan Bautista Avalle Arce, Daniel Eisenberg, Federico F. Curto Herrero, Sylvia Roubaud, Lilia Ferrario de Orduna o Juan Manuel Cacho Blecua han dado paso a una nómina casi inabarcable de investigadores y de estudios en los últimos veinte años, lo que ha propiciado una revisión -casi completa- del género que se abre a nuevas perspectivas de análisis de la mano de las corrientes

\footnotetext{
' Daniel Eisenberg y M." Carmen Marin Pina, Bibliografia de los libros de caballerías castellanos, Zaragoza, Prensas Universitarias, 2000.
}

RFE, LXXXII, $2002,33^{\circ}-4 .^{\circ}$, págs. $407-419$ 
críticas más innovadoras, como la narratología, a la que el estudio de la ficción caballeresca todavía puede ofrecer muy buenos resultados. El interés que los libros de caballerías despierta entre la comunidad científica se aprecia en las sesiones caballerescas, cada vez más numerosas, de los últimos congresos de la Asociación Internacional Siglo de Oro (1999), de la Asociación Internacional de Hispanistas (julio del 2001) o de la Asociación Hispánica de Literatura Medieval (septiembre del 2001), sin olvidar los congresos y seminarios que han tenido a los libros de caballerías como tema central: XXI Edad de Oro: "Libros de caballerias: textos y contextos", celebrado en la Universidad Autónoma de Madrid y en Cuenca (Marzo del 2001) o De los libros de caballerias al "Quijote", que se impartió en Salamanca dentro de las actividades del SEMYR durante los primeros días del mes de junio del 2001, por sólo citar dos de los más recientes.

La información se multiplica, se hace para ciertos textos dificil de controlar; por esta razón son de agradecer los estados de la cuestión publicados en los últimos años por M. ${ }^{a}$ Carmen Marín Pina ("La literatura caballeresca. Estado de la cuestión. 2. Los libros de caballerías españoles", Romanistisches Jahrbuch, 46 (1995), págs. 314-338) y por Anna Bognolo ("Las novelas de caballerias (1995-1999)", en Christoph Strosetzki, Actas del $V$ Congreso de la Asociación Internacional Siglo de Oro, Vervuert, Iberoamericana, 2000, págs. 215-238). El profesor Juan Manuel Cacho Blecua desde la Universidad de Zaragoza está ultimando, junto a un equipo de investigadores de varias universidades, una base de datos, tanto bibliográfica como textual, de la materia caballeresca, que podrá ser consultada por Internet, lo que supondrá un paso de gigante en la recepción del género.

Dentro de este contexto de revitalización de los estudios caballerescos tanto dentro como fuera de España, resulta de una enorme utilidad la reciente Bibliografia de los libros de caballerias castellanos de Daniel Eisenberg y M. ${ }^{a}$ Carmen Marín Pina, que es algo más que una segunda edición de la publicada en 1979, tal y como afirman en un exceso de modestia sus autores. Las más de dos mil entradas bibliográficas de la que consta permiten comprender la envergadura del proyecto; pero, junto a su valor cuantitativo y acumulativo (lo que justificaría ya una obra de estas características), la Bibliografia de Eisenberg y Marín Pina está organizada en diferentes apartados que la convierten en una herramienta extremadamente útil, ya que, además de los estudios y ediciones específicas de cada obra -ordenadas en el libro de manera alfabética - se han incorporado dos secciones iniciales: "Fuentes bibliográficas" (con 96 entradas) ${ }^{2}$ y "Obras

\footnotetext{
2 "Esta sección incluye las fuentes bibliográficas generales más importantes que hemos tenido ocasión de consultar durante la redacción de la presente obra. Se han incorporado trabajos
} 
generales" (con 526), así como diferentes índices, que permiten acceder a la información desde muy variadas perspectivas: [1] Bibliotecas. [2] Impresores y libreros. [3] Libros de caballerías impresos: ediciones perdidas o en paradero desconocido. [4] Libros de caballerías impresos: cronología de primeras ediciones. [5] Libros de caballerías manuscritos. [6] Libros de caballerías microfilmados o reproducidos en facsímil. [7] Libros de caballerías perdidos. [8] Lugares de edición hasta 1623. [9] Onomástico. [10] Temático. Pero sobre todos estos aspectos, hay uno que merece un especial aplauso: la organización cronológica de las entradas bibliográficas, tanto en los apartados generales como en los dedicados a cada texto, distinguiendo claramente entre ediciones y estudios. El índice onomástico permite (con un pequeño esfuerzo) acceder a los estudios de un determinado investigador al margen del tiempo. La ordenación cronológica convierte la Bibliografia de los libros de caballerías castellanos de Daniel Eisenberg y M.a Carmen Marín Pina en' una excelente radiografia de los estudios caballerescos a través del tiempo. Permite comprobar - con sólo una consulta superficial - cuándo se han estudiado más y mejor los textos caballerescos, o qué temas son los que predominan en un momento determinado.

Una obra como ésta, con sus más de dos mil entradas bibliográficas, es la mejor demostración de lo mucho que se ha avanzado en el conocimiento de la materia caballeresca, no cabe duda; miles de páginas se han escrito y publicado. Pero tampoco nos dejemos engañar por los números y las cifras, ya que en la radiografia de la materia caballeresca que podemos extraer de la Bibliografia hay también argumentos para el pesimismo. Del total de 58 textos conservados que han entrado en la nómina del libro ${ }^{3}$, sólo nueve superan la veintena de acercamientos críticos hasta el 2000:

1. Amadis de Gaula (I-IV): 595

2. Sergas de Esplandián (libro v de Amadis de Gaula): 73

3. Lisuarte de Grecia de Feliciano de Silva (libro VII de Amadis de Gaula): 30

\footnotetext{
que, aunque ya han sido superados, tienen interés histórico para el coleccionismo y para el conocimiento de los libros de caballerías castellanos. No registramos, en cambio, los muchos catálogos de bibliotecas revisados ni los referidos a colecciones de libros de interés general para el hispanismo", pág. 15.

${ }^{3}$ No tenemos en cuenta los textos perdidos o dudosos: [1: $\left.\mathrm{n}^{\circ} 623\right]$ libro escrito por Teresa de Ahumada y Cepeda, futura Santa Teresa de Jesús. [2: $\mathrm{n}^{\circ}$ 1504] "Tercera parte de la cuarta de Florisel de Niquea". [3: $\mathrm{n}^{\circ}$ 1533]: posible continuación cervantina del Belianís de Grecia. [4: $\mathrm{n}^{\circ}$ 1545] Miguel de Cervantes, Bernardo del Carpio. [5: $\left.\mathrm{n}^{\circ} 1551\right]$ Caballero de la Rosa. [6: $\mathrm{n}^{\circ}$ 1602] Libros $\vee$ y $\mathrm{Vl}$ de Clarián de Landanis. [7: $\mathrm{n}^{\circ}$ 1782]: Florimón. [8: $\mathrm{n}^{\circ}$ 1796] Román Ramírez, Florisdoro de Grecia. [9: $\mathrm{n}^{\circ}$ 1810] Leoneo de Hungria. [10: $\mathrm{n}^{\circ}$ 1812] Leonís de Grecia. [11: $\mathrm{n}^{\circ}$ 2029] Joaquín Romero de Cepeda, Rosián de Castilla. [12: $\mathrm{n}^{\circ}$ 2039] Taurismundo.
} 
4. Amadis de Grecia de Feliciano de Silva (libro IX de Amadis de Gau(a): 22

5. Claribalte de Gonzalo Fernández de Oviedo: 23

6. Espejo de principes y caballeros (I) de Diego Ortúñez de Calahorra; 35

7. Palmerín de Olivia de ¿Francisco Vázquez?: 63

8. Primaleón de ¿Francisco Vázquez?: 36

9. Tristán el Joven: 21

Y sobre ellos, sobresale el emperador de la caballería, el Amadis de Gaula que sigue siendo el texto caballeresco más estudiado, el que termina por convertirse en paradigma de todo un género, en donde confluyen miles de matices y páginas.

$Y$ las cifras son todavía más descorazonadoras cuando nos adentramos en el resto del corpus caballeresco: catorce títulos, a pesar de pertenecer a ciclos tan importantes como el amadisiano, no llegan ni a estas cifras:

1. Florisando (libro $\mathrm{V}$ de Amadís de Gaula): 8

2. Florisel de Niquea de Feliciano de Silva (III): 6

3. Florisel de Niquea de Feliciano de Silva (IV): 11

4. Silves de la Selva de Pedro de Luján (libro XII de Amadís de Gaula): 6

5. Belianis de Grecia (I-II) de Jerónimo Fernández: 19

6. Cirongilio de Tracia de Bernardo de Vargas: 5

7. Clarián de Landanís (I, libro I) de Gabriel Velázquez de Castillo: 6

8. Clarián de Landanis (I, libro II) de Álvaro de Castro: 6

9. Clarisel de las Flores de Jerónimo Jiménez de Urrea: 8

10. Cristalián de España de Beatriz Bernal: 15

11. Florindo de Fernando Basurto: 8

12. Floriseo de Fernando Bernal: 6

13. Lidamarte de Armenia de Damasio de Frías y Balboa: 8

14. Olivante de Latra de Antonio de Torquemada: 10

Pero aún podemos dar otra vuelta de tuerca al pesimismo, ya que más de treinta títulos siguen hoy en día sin estudios, o con el acercamiento aislado de un crítico, en ocasiones en análisis globales del género:

1. Lisuarte de Grecia de Juan Díaz (libro VIII de Amadis de Gaula): 2

2. Florisel de Niquea de Feliciano de Silva (1-II): 2

3. Belianis de Grecia (III) de Jerónimo Fernández: 0

4. Belianis de Grecia (IV) de Pedro Guiral de Verrio: 1

5. Bencimarte de Lusitania: 1

6. Caballero de la Luna: 1

7. Floramante de Colonia (parte Il de Clarián) de Jerónimo López: 1

8. Lidamán de Ganail (parte IV de Clarián) de Jerónimo López: 2

9. Claridoro de España: 1

10. Claris de Trapisonda: 1

11. Espejo de principes y caballeros (II) de Pedro de la Sierra Infanzón: 2 
12. Espejo de principes y caballeros (III) de Marcos Martínez: 3

13. Espejo de principes y caballeros ([IV]-V): 1

14. Febo el Troyano de Esteban de Corbera: 2

15. Félix Magno: 3

16. Felixmarte de Hircania de Melchor Ortega: 4

17. Filesbián (o Philesbián) de Candaria: 4

18. Filorante: 1

19. Flor de caballerias: 2

20. Florambel de Lucea (I) de Francisco de Enciso: 1

21. Florambel de Lucea (II) de Francisco de Enciso: 0

22. Florambel de Lucea (III) de ¿Francisco de Enciso?: 1

23. Florando de Inglaterra (II): 2

24. Reimundo de Grecia (libro III de Floriseo): 0

25. Leon Flos de Tracia: 1

26. Lepolemo de Alonso de Salazar: 3

27. Lidamor de Escocia de Juan de Córdoba: 2

28. Marsindo: 2

29. Mexiano de la Esperanza: 2

30. Platir de Jerónimo de Enciso: 2

31. Policisne de Boecia de Juan de Silva y de Toledo: 3

32. Polindo: 3

33. Polismán de Jerónimo de Contreras: 3

34. Roselao de Grecia (libro III de Espejo de caballerias): 1

35. Valerián de Hungria de Dionís Clemente: 1

Más de cincuenta textos diferentes, pero ¿a cuántos tenemos acceso gracias a ediciones modernas? $\mathrm{Si}$ dejamos a un lado las 24 ediciones (completas y fragmentarias) de los cuatro primeros libros de Amadis de Gaula, la situación del resto de los libros resulta especialmente descorazonadora: o se trata de ediciones publicadas en los años sesenta y setenta, actualmente descatalogadas, como la edición del Palmerín de Olivia, realizada por Giuseppe di Stefano (Pisa, Universitá, 1966) o la del Espejo de príncipes y caballeros de Diego Ortúñez de Calahorra, que llevó a cabo Daniel Eisenberg (Madrid, Espasa-Calpe, 1975 [1976]), o se trata de ediciones aparecidas en los últimos años, en colecciones que, en ocasiones, son de dificil adquisición o consulta: los primeros libros de $B e$ lianís de Grecia de Jerónimo Fernández, editados por Lilia Ferrario de Orduna (Kassel, Reichenberger, 1997), el Clarián de Landanis (iibro I) de Gabriel Velázquez de Castillo, por G. Anderson (Newark, Juan de la Cuesta, 1995), el Olivante de Laura de Antonio de Torquemada, por María Isabel Muguruza (Madrid, Ediciones Castro, 1997) o el Tristán el Joven, por Luzdivina Cuesta Torre (México, UNAM, 1997). Sin textos, los estudios son escasos. Un circulo vicioso que ha querido romper el Centro de Estudios Cervantinos (Alcalá de Henares) con un ambicioso proyecto editorial: Los libros de Rocinante, que se propone la edición del 
corpus completo de los libros de caballerías. Hasta el año 2001, se han publicado once títulos:

1. Platir, ed. M. ${ }^{a}$ Carmen Marín Pina (1997).

2. Flor de caballerias de Francisco de Barahona, ed. José Manuel Lucía Megías (1997).

3. Primaleón, ed. M. ${ }^{a}$ Carmen Marín Pina (1998).

4. Felixmarte de Hircania de Melchor Ortega, ed. de Rosario Aguilar Perdomo (1998).

5. Tercera parte de Florisel de Niquea de Feliciano de Silva, ed. de Javier Martín Lalanda (1999).

6. Tristán de Leonis, ed. Luzdivina Cuesta (1999).

7. Arderique, ed. Dorothy A. Carpenter (2000).

8. Clarián de Landanis (libro II), ed. de Javier Guijarro Ceballos (2000).

9. Félix Magno (I-II), ed. Claudia Demattè (2001).

10. Félix Magno (III-IV), ed. de Claudia Demattè (2001).

11. Claribalte de Gonzalo Fernández de Oviedo, ed. Alberto del Rio Nogueras (2001).

Con esta misma finalidad de dar a conocer el complejo entramado textual caballeresco, como un medio de potenciar el estudio de otros textos al margen del Amadis de Gaula, el Centro de Estudios Cervantinos publicó en el 2001 una Antologia de los libros de caballerías castellanos coordinada por mí mismo, así como sigue adelante con su colección Guias de lectura caballeresca, en donde ofrece informaciones particulares (argumento, diccionario y listado de personajes y bibliografia) de cada uno de los libros de caballerías castellanos; hasta el 2001 se han publicado un total de 22 títulos ${ }^{4}$. Esperemos que estas propuestas hagan posible cambiar el reparto de cifras

\footnotetext{
${ }^{4}$ [1] Amadis de Grecia de Feliciano de Silva $\left(\mathrm{n}^{\circ} 26\right)$ por Carmen Laspuertas: 2001; [2] Anderique ( $\mathrm{n}^{\mathrm{0}}$ 11) por Dorothy Carpenter: 2000. [3] Baladro del sabio Merlin (1498) ( $\mathrm{n}^{\circ} 1$ ) por Paloma Gracia: 1999. [4] Baldo (Sevilla, 1542) ( $\mathrm{n}^{\circ} 38$ ) por Folke Gernert: 2000. [5] Cirongilio de Tracia $\left(n^{\circ} 41\right)$ por Javier Roberto González: 2000. [6] Clarián de Landanís (Primera parte. Libro primero) ( $\mathrm{n}^{\circ}$ 12) por Antonio Joaquín González Gonzalo: 1998. [7] Claribalte de González Fernández de Oviedo $\left(\mathrm{n}^{\circ}\right.$ 13) por Alberto del Rio Nogueras, 2001. [8] Libro segundo de don Clarián de Landanís ( $\mathrm{i}^{\circ}$ 16) por Javier Guijarro Ceballos: 2000. [9] Espejo de príncipes y caballeros (parte II) $\left(\mathrm{n}^{\circ}\right.$ 60) por José Julio Martín: 2001. [10] Felixmarte de Hircania ( $\mathrm{n}^{\mathrm{n}}$ 55) por María del Rosario Aguilar Perdomo: 1998. [11] Félix Magno (libros 1-II) $\left(\mathrm{n}^{\circ} 40\right)$ por Claudia Demattè: 2000. [12] Félix Magno (iibros III-IV) $\left(\mathrm{n}^{\circ} 51\right)$ por Claudia Demattè: 2001. [13] Flarando de Inglaterra (I-1]) $\left(\mathrm{n}^{\circ} 43\right)$ por Cristina Castillo: 2001. [14] Florisando de Páez de Ribera ( $\mathrm{n}^{\circ}$ 5) por Cristina Ramos: 2001. [15] Florisel de Niquea (parte III) ( $\mathrm{n}^{\circ}$ 45) por Javier Martin Lalanda: 1999. [16] Floriseo ( $\left.\mathrm{n}^{\circ} 10\right)$ por Javier Gujjarro Ceballos: 1999. [17] Lidamor de Escocia $\left(\mathbf{n}^{\circ}\right.$ 34): por Jorge Francisco Sáenz Carbonell: 1999. [18] Oliveros de Castilla $\left(n^{\circ}\right.$ 2) por José Manuel Lucía Megías: 1998. [19] Sergas de Esplandian $\left(\mathrm{n}^{\circ}\right.$ 14): por Emilio J. Sales Dasí: 1999. [20] Lisuarte de Grecia ( $\mathrm{n}^{\circ}$ 19): por Emilio J. Sales Dasí: 1998. [21] Tristán de Leonis ( $\mathrm{n}^{\circ} 3$ ) por Luzdivina Cuesta Torre: 1998. [22] Tristán de Leonís el Joven $\left(\mathrm{n}^{\circ} 35\right)$ por Luzdivina Cuesta Torre: 1999.
} 
de los estudios caballerescos en una segunda edición de la Bibliografia de los libros de caballerias castellanos de Daniel Eisenberg y M. ${ }^{*}$ Carmen Marín Pina, o en la citada base de datos electrónica dirigida por Juan Manuel Cacho Blecua.

En esta radiografía del género caballeresco que constituye la bibliografia citada, se aprecia una de las labores más apremiantes en el conjunto del género: el establecimiento de un corpus de obras que deben englobarse dentro del epígrafe de "libros de caballerías castellanos". El género caballeresco se difunde en los siglos xVI y XVII en una maraña textual de gran complejidad: desde las modalidades más populares (los pliegos de cordel a las historias caballerescas breves) a las más cultas (libros de caballerías en verso); desde los textos en folio de grandes dimensiones (libros de caballerias) a sus transformaciones más específicas, como los libros de caballerías a lo divino. $\mathrm{Y}$ todo ello, por centrarnos en el campo textual, ya que obras de teatro, fiestas caballerescas, comportamiéntos cortesanos o modelos iconográficos podrían también englobarse en la recepción y difusión coetánea de este universo durante los citados siglos.

A Pascual de Gayangos y a José Ignacio Ferreras debemos los primeros intentos de establecer un corpus de libros de caballerías castellanos. En ambos casos, su criterio se basó exclusivamente en el contenido. Así se expresaba Gayangos en su Catálogo razonado de los libros de caballerias que hay en lengua castellana ó portuguesa hasta el año 1800 (Madrid, Rivadeneyra, 1857): "Para tratar de estos libros con el debido órden, convendrá dividirlos en tres grandes ciclos: el breton, el carlovingio y el greco-asiático. Los dos primeros son, con alguna ligera excepción, exclusivamente franceses; y el tercero fué engendrado en la Península por la brillante imaginación de nuestros escritores. A este último habrá necesariamente de agregarse otra multitud de libros, así en prosa como en verso que, estrictamente hablando, no son más que una modificación del género, como son la novela-caballeresca-sentimental, los libros de caballerías morales ó á lo divino, los que están fundados sobre la historia de España, y por último, las bellísimas epopeyas caballerescas traducidas ó imitadas del italiano" (pág. vi). Mucho más generoso en los límites cronológicos (se incluyen obras medievales) como lingüísticos (no sólo obras en castellano, sino también en portugués) se ofrece la clasificación de Juan Ignacio Ferreras ${ }^{5}$, que, basándose en la clasificación medieval de Jean Bodel ("Materia de Roma, de Francia y de Bretaña") a la que añade una "Materia castellana", lleva a incluir en el mismo

\footnotetext{
5 "La materia castellana en los libros de caballerías (Hacia una nueva clasificación", Philologica Hispaniensia in honorem Manuel Alvar, tomo III: Literatura, Madrid, Gredos, 1986, págs. 121-141.
} 
rótulo caballeresco libros de caballerías a lo divino (El caballero del sol de Pedro Hernández de Villaumbrales, La caballería celestial de la Rosa Fragrante de Jerónimo de San Pedro, la Caballería Cristiana de Fray Jaime de Alcalá, o la Historia y milicia cristiana del caballero Peregrino de Fray Alonso de Soria) así como poemas caballerescos (Celidón de Iberia de Gómez de Luque, y otra obra con el mismo título de Francisco de Sandoval).

La aparición en 1979 del Castilian Romances of Chivalry in the Sixteenth Century: A Bibliography de Daniel Eisenberg, como ya hemos indicado, vino a poner un poco de luz a este corpus caballeresco que, a pesar de los enormes esfuerzos científicos indicados (o el que lleva a cabo José Simón Díaz en el tomo III de su monumental Bibliografia de la Literatura Hispánica, Madrid, CSIC, 1953) todavia se movía en las arenas movedizas de la imprecisión. Pero, a pesar de sus grandes virtudes, el problema del establecimiento de un corpus, y la explicación del mismo, se dejó en un segundo plano; ya que los límites para establecer qué textos formarian parte del catálogo se concretaron en tres puntos, básicamente: [1] el límite cronológico se estableció entre 1508 y 1602, aunque en el caso de Espejo de príncipes y caballeros se amplió hasta 1623; [2] se suprimieron las traducciones: "deliberately excluded are indentifiable translations from other languages"; y [3] se omitieron Ios textos artúricos, ya que éstos habian sido el objeto de un catálogo similar realizado por Harvey $\mathrm{L}$. Sharrer en la editorial londinense Grant \& Cutler. Lamentablemente, una situación similar se reproduce en la excelente Bibliografia de los libros de caballerias castellanos (2000), en donde la explicación de los limites del corpus - -aspecto todavía no clarificado por la crítica- se despacha con el siguiente párrafo: "La obra se limita a Amadís de Gaula y a los posteriores libros de caballerías escritos originariamente en castellano. Excluimos, por tanto, Tirante el Blanco, Palmerín de Inglaterra, o los primeros libros de Espejo de caballerías. No nos ocupamos tampoco del medieval Caballero Cifar, aunque se publicó a comienzos del siglo xvı al calor del éxito editorial de los nuevos libros de caballerías para abastecer las peticiones del público, ni de traducciones como la Demanda del santo Grial. Como ejemplo del progreso alcanzado en el estudio de los libros de caballerías durante los últimos veinte años, en esta nueva edición se ha suprimido Arderique, sólo ahora reconocido como traducción" (pág. 9). El criterio para establecer los límites del corpus de los libros de caballerías va más allá del contenido, centrándose en el hecho de que fueran escritos originariamente en castellano.

En el 2000 propuse un corpus de los libros de caballerías castellanos en Imprenta y libros de caballerias (Madrid, Ollero \& Ramos), al que quisiera volver de nuevo. En aquella ocasión, como posteriormente en la 
Antología de libros de caballerías castellanos (Alcalá de Henares, Centro de Estudios Cervantinos, 2001) indicaba la necesidad de conjugar varios criterios a la hora de establecer el corpus de los libros de caballerías; criterios que intentaran rescatar el marco coetáneo de recepción de este género caballeresco, que era fácilmente reconocible por los lectores de su época; no hay nada más que releer algunas de las críticas que se publicaron en el siglo XVI, en donde, con hacer alusión a unos pocos títulos, tanto el autor como el lector estarían asumiendo un número mayor de textos, así como en apreciaciones sobre su formato, como la que les lleva al cura y al barbero cervantino a distinguir por su tamaño los libros de caballerías de otros géneros en la biblioteca del hidalgo Alonso Quijano. En resumen, se proponía tener en cuenta tanto su contenido (su naturaleza literaria, es decir el texto) como su forma exterior de transmisión (su naturaleza editorial, es decir el libro). Sólo conjugando esta doble perspectiva, sería posible entender en su complejidad un género que pervive a lo largo del siglo xvi y en los primeros decenios del xvII tanto por la capacidad de adaptar sus contenidos a los nuevos horizontes de expectativas del público (en una clara línea de evolución que va de los modelos idealistas - Amadis de Gaula - a los de entretenimiento - Espejo de principes y caballeros y Don Quijote de la Mancha-) como por ser un exitoso producto comercial que permitió a muchas imprentas hispánicas competir con otras europeas, que gozaban del monopolio y de los ingresos del tlamado libro internacional. Desde esta perspectiva, el corpus de los libros de caballerías castellanos sólo tiene que dejar a un lado aquellos textos que, desde una clara estrategia comercial, se visten de los ropajes editoriales de la caballería para aprovecharse de un público potencial, siendo su naturaleza literaria completamente distinta, como sucede con la impresión del texto medieval del Libro del caballero Cifar que sale a la luz en las prensas sevillanas de Jacobo Cromberger en 1512, o de las diferentes crónicas reales que a mediados del siglo XVI se editan en Medina del Campo con el mismo formato y grabado con jinete en portada que los libros de caballerías que se imprimen de manera contemporánea. Desde esta perspectiva la lengua original -lo que, en algunos casos, sólo sabemos en la actualidad, como ha sucedido con el Arderique- no puede ser un criterio dirimente para dejar fuera del corpus de los libros de caballerías castellanos algunos de los textos más influyentes de su época; textos que aparecen en los inventarios de libros, en la nómina de personajes de otros títulos caballerescos, en las críticas al género. Si dejamos fuera el Tirante el Blanco, terminado de publicar por Diego de Gumiel en su taller vallisoletano el 28 de mayo de 1511, ¿cómo entender algunos textos valencianos publicados en años posteriores que destacan por sus detalles 
realistas, seguramente influidos por el texto castellano del Tirant, como son el Floriseo (1516), el Arderique (1517), el Claribalte (1519) o el Lepolemo (1521)? ¿Cómo dejar fuera del corpus de libros de caballerías las dos primeras entregas del Espejo de caballerias de Pedro López de Santa Catalina, publicados en Toledo en 1525 y 1527, y sí incluir el tercer libro del ciclo, el que Pedro de Reinosa escribe y se publica en el taller toledano de Juan de Ayala en 1547? ¿Acaso la traducción castellana del Palmerín de Inglaterra, del ciclo de Renaldos de Montalbán o los ejemplos citados del Espejo de caballerias no ofrecen textos, parcialmente nuevos, adaptados al público castellano que, y eso es lo importante, son recibidos como textos tan originales o tan traducidos como el resto, como el propio Amadis de Gaula?

Desde este doble criterio, el que tiene en cuenta las dos caras del libro de caballerías castellano, que es a un tiempo portador de un universo literario y ejemplo de un producto editorial que mantiene, inalterables, unas características externas a lo largo de su larga existencia, el corpus de los libros de caballerías castellanos podría estar formado por los siguientes setenta y cinco títulos. A los impresos, se les han añadido los textos manuscritos que, aunque no entran dentro del mundo de la imprenta, muestran una difusión y una pervivencia del género más allá de lo que las empobrecidas prensas hispánicas pudieran hacer pensar a finales del siglo XVI. Los libros de caballerías castellanos se explican en muchos de sus matices cuando se asume que representan uno de los géneros editoriales más significativos de la centuria ${ }^{6}$ :

[1] Adramón [MS]

[2] Amadis de Gaula (I-IV) de Garci Rodríguez de Montalvo

[3] Las sergas de Esplandián (v) de Garci Rodríguez de Montalvo

[4] Florisando (VI) por Ruy Páez de Ribera

[5] Lisuarte de Grecia (VII) de Feliciano de Silva

[6] Lisuarte de Grecia (VIII) de Juan Díaz

[7] Amadis de Grecia (IX) de Feliciano de Silva

[8] Florisel de Niquea (X: partes I-II) de Feliciano de Silva

[9] Florisel de Niquea (XI: parte III) de Feliciano de Silva

[10] Florisel de Niquea (XI: parte IV) de Feliciano de Silva

\footnotetext{
${ }^{6}$ Con una flecha $(\rightarrow)$, se indican aquellos titulos que no han entrado en la Bibliografia de los libros de caballerias castellanos de Daniel Eisenberg y M.' Carmen Marin Pina (2000). Con [MS], se identifican los libros de caballerias manuscritos dentro del corpus. Un repertorio completo de ediciones antiguas y ejemplares conservados, puede consultarse en la citada bibliografia, así como en la Antologia de libros de caballerias castellanos, ob. cit, y en mi artículo: "Libros de caballerías castellanos: textos y contextos", Edad de Oro, 14 (2002).
} 
[11] Silves de la Selva (XII) de Pedro de Luján

[12] Arderique $(\rightarrow)$

[13] Baladro del sabio Merlin $(\rightarrow)$

[14] Demanda del santo Grial $(\rightarrow)$

[15] Belianís de Grecia (partes I-II) de Jerónimo Fernández

[16] Belianís de Grecia (partes III-IV) de Jerónimo Fernández

[17] Belianis de Grecia (parte v) de Pedro Guiral de Verrio [MS]

[18] Bencimarte de Lusitania [MS]

[19] Caballero de la Luna (libros III-IV) [MS]

[20] Cirongilio de Tracia de Bernardo de Vargas

[21] Clarián de Landanis (primera parte, libro I) de Gabriel Velázquez de Castillo

[22] Clarián de Landanis (primera parte, libro II) de Álvaro de Castro

[23] Floramante de Colonia (segunda parte de Clarián de Landanis) de Jerónimo López

[24] Clarián de Landanis (libro III) de Jerónimo López

[25] Lidamán de Ganail (cuarta parte de Clarián de Landanis) de Jerónimo López

[26] Claribalte de Gonzalo Fernández de Oviedo

[27] Claridoro de España [MS]

[28] Claris de Trapisonda [MS]

[29] Clarisel de las Flores de Jerónimo Jiménez de Urrea [MS]

[30] Cristalián de España de Beatriz Bernal

[31] Espejo de caballerías (libro I) de Pedro López de Santa Catalina $(\rightarrow)$

[32] Espejo de caballerías (libro II) de Pedro López de Santa Catalina $(\rightarrow)$

[33] Don Roselao de Grecia (libro III de Espejo de caballerias) de Pedro de Reinosa

[34] Espejo de principes y caballeros (I) de Diego Ortúñez de Calahorra

[35] Espejo de principes y caballeros (II) de Pedro de la Sierra

[36] Espejo de principes y caballeros (III[-IV]) de Marcos Martínez

[37] Espejo de principes y caballeros (V) [MS]

[38] Febo el Troyano de Esteban Corbera

[39] Félix Magno (libros I-IV)

[40] Felixmarte de Hircania de Melchor Ortega

[41] Filorante [MS]

[42] Flor de caballerias de Francisco de Barahona [MS]

[43] Florambel de Lucea (partes I-II) de Francisco de Enciso Zárate

[44] Florambel de Lucea (parte III) de Francisco de Enciso Zárate [MS]

[45] Florando de Inglaterra

[46] Florindo de Fernando Basurto

[47] Floriseo (libros I-II) de Fernando Bernal 
[48] Reimundo de Grecia (libro III de Floriseo) de Fernando Bernal

[49] Guarino Mezquino $(\rightarrow)$

[50] Leon Flos de Tracia [MS]

[51] Lepolemo (El Caballero de la Cruz) de Alonso de Salazar

[52] Leandro el Bel $(\rightarrow)$

[53] Lidamarte de Armenia de Damasio de Frias y Balboa [MS]

[54] Lidamor de Escocia de Juan de Córdoba

[55] Marsindo [MS]

[56] Mexiano de la Esperanza (primera parte) de Miguel Daza [MS]

[57] Morgante de Jerónimo Aunés ( $\rightarrow$ )

[58] Olivante de Laura de Antonio de Torquemada

[59] Oliveros de Castilla $(\rightarrow)$

[60] Palmerin de Inglaterra $(\rightarrow)$

[61] Palmerin de Olivia de ¿Francisco Vázquez?

[62] Primaleón de ¿Francisco Vázquez?

[63] Platir de Francisco de Enciso Zárate

[64] Philesbián de Candaria

[65] Policisne de Boecia de Juan de Silva y Toledo

[66] Polindo

[67] Polismán de Jerónimo de Contreras [MS]

[68] Renaldos de Montalbán (libros I-II) de Luis Domínguez $(\rightarrow)$

[69] La Trapesonda (libro III de Renaldos de Montalbán) ( $\rightarrow$ )

[70] Baldo (libro IV de Renaldos de Montalbán) $(\rightarrow)$

[71] Selva de Cavalarias (segunda parte) de Antonio de Brito da Fonseca $(\rightarrow)$ [MS]

[72] Tirante el Blanco $(\rightarrow)$

[73] Tristán de Leonis $(\rightarrow)$

[74] Tristán el Joven

[75] Valerián de Hungria de Dionís Clemente

La excelente y útil herramienta que Daniel Eisenberg y M. ${ }^{a}$ Carmen Marín Pina han puesto a disposición de todos los investigadores permite comprobar todo lo que se ha hecho hasta este momento en este campo, especialmente en los últimos años; pero también pone delante de nuestros ojos, con una claridad cegadora, todo lo que queda por hacer, que es mucho. Mucho para dar a conocer los textos; mucho para poder comprender y asimilar los mil matices que albergan, un verdadero laboratorio de experimentación narrativa, del que podrian aprender bastantes escritores actuales; mucho hasta llegar a síntesis criticas y estudios globales del género, que no se ha intentado desde el libro de sir Henry Thomas Spanish and Portuguese Romances of Chivalry. The revival of the Romance of Chivalry in the Spanish Peninsula 
and its Extension and Influence Abroad (Cambridge, Cambridge University Press, 1920$)^{7}$; mucho, incluso, para precisar conceptos e ideas básicas, como he querido exponer en estas páginas, ya que algunas preguntas preliminares aún no han sido contestadas de manera definitiva por la crítica, como el establecimiento de un corpus de obras que se engloben bajo el epígrafe de "libros de caballerías castellanos". En todo caso, herramientas como la Bibliografia de libros de caballerias castellanos de Daniel Eisenberg y $\mathrm{M}^{\mathrm{a}}$ Carmen Marin Pina suponen un gran paso adelante en el estudio de la materia caballeresca. Un gran paso adelante que nos anima a seguir trabajando en un campo cada vez más apasionante, cada vez más rico, cada vez más lleno de sorpresas y de respuestas en un momento crucial para la creación de la narrativa moderna; un paso que permite mirar los libros de caballerías castellanos en el siglo XXI con nuevos ojos, sin las vendas que la crítica se impuso desde el siglo XVIII con la finalidad de separar el Quijote del género que le vio nacer y sobre el que se levantó el genio de Cervantes que es, sin duda, uno de los mejores autores de libros de caballerías. ¿Acaso no ha llegado el momento de incluir el Quijote dentro del corpus de los libros de caballerías castellanos, el momento de devolverle su naturaleza textual y editorial?

' Reimpreso en 1969 (Nueva York, Kraus Reprint) y traducido al español por Esteban Pujais: Las novelas de caballerias españolas y portuguesas. Despertar de la novela caballeresca en la Peninsula Ibérica y expansión e influencia en el extranjero. Anejos de la Revista de Literatura, 10, Madrid, CSIC, 1952. 